\title{
Effects of beraprost sodium on renal function and inflammatory factors of rats with diabetic nephropathy
}

\author{
J. Guan ${ }^{1,2}$, L. Long', Y.-Q. Chen ${ }^{1}$, Y. Yin ${ }^{1}$, L. Li ${ }^{1}$, C.-X. Zhang ${ }^{1}$, \\ L. Deng ${ }^{1}$ and L.-H. Tian ${ }^{1}$ \\ ${ }^{1}$ Affiliated Hospital of North Sichuan Medical College, Nanchong, Sichuan, \\ China \\ ${ }^{2}$ Nursing School of North Sichuan Medical College, Nanchong, Sichuan, China \\ Corresponding author: J. Guan \\ E-mail: guanji_guanji@yeah.net /35438428@qq.com
}

Genet. Mol. Res. 13 (2): 4154-4158 (2014)

Received November 19, 2012

Accepted November 13, 2013

Published June 9, 2014

DOI http://dx.doi.org/10.4238/2014.June.9.1

\begin{abstract}
Beraprost sodium (BPS) is a prostaglandin analogue. We investigated its effects on rats with diabetic nephropathy. There were 20 rats each in the normal control group (NC), the diabetic nephropathy group (DN), and the BPS treatment group. The rats in DN and BPS groups were given a high-fat diet combined with low-dose streptozotocin intraperitoneal injections. The rats in the BPS group were given daily $0.6 \mathrm{mg} / \mathrm{kg}$ intraperitoneal injections of this drug. After 8 weeks, blood glucose, 24-h UAlb, Cr, BUN, hs-CRP, and IL-6 levels increased significantly in the DN group compared with the NC group; however, the body mass was significantly reduced in the DN group compared with the NC group. Blood glucose, urine output, 24-h UAlb, $\mathrm{Cr}$, hs-CRP, and IL-6 levels were significantly lower in the BPS group than in the DN group; the body mass was significantly greater in the DN group. Therefore, we concluded that BPS can improve renal function and protect the kidneys of DN rats by reducing oxidative stress and generation of inflammatory cytokines; it also decreases urinary protein
\end{abstract}


excretion of rats with diabetic nephropathy.

Key words: Beraprost sodium; Diabetic nephropathy; Renal function; Inflammatory factors

\section{INTRODUCTION}

Diabetic nephropathy is a major microvascular complication of diabetes, its pathogenesis is very complicated and it is not fully elucidated. Many studies have confirmed that oxidative stress plays an important role in the pathogenesis of diabetic nephropathy. High blood glucose can cause increased production of reactive oxygen species, the activation of the polyol and hexosamine pathways, and high levels of protein kinase $\mathrm{C}$ and advanced glycosylation products, where all these factors participate in the development of diabetic nephropathy (Brownlee, 2005). Some studies have reported that beraprost sodium (BPS) is a vasoactive substance that can expand renal vessels, increase renal blood flow, inhibit TXA2 synthesis, platelet aggregation and immune complex formation, and prevent glomerular thrombosis to finally reduce proteinuria (Gryglewski, 2008) In this study, we used a high-fat diet combined with intraperitoneal injection of low-dose streptozotocin (STZ) $(30 \mathrm{mg} / \mathrm{kg})$ to establish the rat model of type 2 diabetes (T2DM). Body mass, kidney function, urinary protein, blood glucose, and other indicators were observed to investigate the efficacy of BPS in diabetic nephropathy and its possible mechanisms.

\section{MATERIAL AND METHODS}

\section{Reagents}

BPS (Dana, Astellas Pharma) and STZ were purchased from Sigma Company (USA).

\section{Animals}

Sixty SPF grade male Sprague-Dawley rats were purchased from the animal center of the North Sichuan Medical College Laboratory. The body weight of the rats was $180 \pm 20$ g. They were fed in a clean environment for 7 days for adaption. They were then randomly divided into the normal control group (NC), the diabetic nephropathy model group (DN) and the BPS treatment group (BPS), with each group consisting of 20 rats. The rats in DN and BPS groups were given the high-fat diet for 4 weeks to induce the insulin-resistance model. Intraperitoneal injection of $30 \mathrm{mg} / \mathrm{kg}$ STZ was given once. After blood glucose was stable for 1 week, random blood glucose tests were performed, and the T2DM model was successful if the random blood glucose was $\geq 16.7 \mathrm{mM}$ on two occasions. The NC and DN rats were given ordinary diet. The BPS rats were given daily $0.6 \mathrm{mg} / \mathrm{kg}$ BPS by intragastric administration. Finally, 20 rats were included in each experimental group. The rats were killed and specimens were taken after 8 weeks of treatment.

\section{Detection indicators and tests}

A 24-h urine was collected from the rats in each group, and the 24-h urinary protein (24-h UAlb) (Coomassie Blue assay) was measured after 8 weeks of treatment. The rats were weighed before being killed, and $4 \mathrm{mg} / \mathrm{kg} 10 \%$ chloral hydrate was used to anesthetize the rats by intraperitoneal injection. The left kidney was rapidly removed and weighed; abdominal 
aortic blood was then obtained and the serum separated. Fasting blood glucose (FBG), serum creatinine $(\mathrm{Cr})$ and blood urea nitrogen $(\mathrm{BUN})$ were tested by the laboratory diagnosis division of North Sichuan Medical College using a Hitachi 7020 automatic biochemical analyzer.

\section{Statistical analysis}

The SPSS 17.0 software was used for the statistical analysis. All continuous variables are reported as means $\pm \mathrm{SD}$. One-way ANOVA was used in the comparison of the groups. The LSD test was used in the pairwise comparisons. $\mathrm{P}<0.05$ indicated statistically significant differences.

\section{RESULTS}

\section{Comparisons of FBG, urine output and other renal function indicators}

After 8 weeks of treatment, the body mass of the DN rats showed a significantly greater decrease than in NC and BPS groups $(\mathrm{P}<0.01)$. Body mass in BPS rats changed little compared with the NC group $(\mathrm{P}>0.05)$. FBG, urine output, kidney weight/body weight ratio (KW/BW), 24-h UAlb, $\mathrm{Cr}$, BUN levels of the rats in the DN group were significantly higher than those in the NC group $(\mathrm{P}<$ 0.01 ). FBG and 24-h UAlb levels in the BPS group were significantly lower than in the DN group (P $<0.01)$. Urine output, $\mathrm{KW} / \mathrm{BW}$ and $\mathrm{Cr}$ in the BPS group were also somewhat decreased $(\mathrm{P}<0.05)$, but there was no significant difference in $\mathrm{BUN}$ level between the two groups $(\mathrm{P}>0.05)$ (Table 1).

Table 1. Comparision between the three groups in renal function.

\begin{tabular}{lccc}
\hline Variables & NC group & DN group & BPS group \\
\hline Blood glucose $(\mathrm{mM})$ & $6.05 \pm 0.55$ & $20.13 \pm 1.02^{*}$ & $15.12 \pm 1.16^{* \Delta}$ \\
24 h UAlb $(\mathrm{mg})$ & $4.67 \pm 1.34$ & $14.14 \pm 1.36^{*}$ & $11.15 \pm 1.03^{* \Delta}$ \\
$\mathrm{Cr}(\mu \mathrm{M})$ & $43.12 \pm 12.11$ & $88.31 \pm 20.35^{*}$ & $64.17 \pm 13.38^{* \Delta}$ \\
BUN $(\mathrm{mM})$ & $11.21 \pm 2.17$ & $24.10 \pm 2.13^{*}$ & $20.11 \pm 2.11^{*}$ \\
Urine output $(\mathrm{mL})$ & $36.11 \pm 10.16$ & $70.16 \pm 17.16^{*}$ & $53.11 \pm 13.22^{* \Delta}$ \\
Body mass $(\mathrm{g})$ & $463.17 \pm 35.84$ & $363.12 \pm 39.23^{*}$ & $434.33 \pm 43.42^{* \Delta}$ \\
\hline NC = normal control group; DN $=$ diabetic nephropathy model group; BPS $=$ BPS treatment group. *P $<0.05$ \\
compared with the NC group. ${ }^{*} \mathrm{P}<0.05$ compared with the DN group.
\end{tabular}

\section{Inflammatory cytokine indicators in rats in different groups}

After 8 weeks of treatment, interleukin-6 (IL-6) and high-sensitivity C-reactive protein (hs-CRP) levels in the DN group were significantly higher than those of the NC group (P $<0.01)$. The hs-CRP and IL-6 levels in the BPS group were significantly lower compared with the DN group $(\mathrm{P}<0.01)$ (Table 2$)$.

Table 2. Comparation between three groups in inflammatory factors.

\begin{tabular}{lrrr}
\hline Variables & NC group & DN group & BPS group \\
\hline IL-6 $(\mathrm{ng} / \mathrm{L})$ & $22.12 \pm 1.45$ & $57.83 \pm 4.55^{*}$ & $41.12 \pm 5.32^{* \Delta}$ \\
hs-CRP $(\mu \mathrm{g} / \mathrm{L})$ & $1.04 \pm 0.21$ & $2.65 \pm 0.10^{*}$ & $1.94 \pm 0.13^{* \Delta}$ \\
TNF- $\alpha(\mathrm{ng} / \mathrm{L})$ & $40.12 \pm 3.22$ & $89.55 \pm 6.95^{*}$ & $66.13 \pm 4.34^{* \Delta}$ \\
\hline
\end{tabular}

${ }^{*} \mathrm{P}<0.05$ compared with the NC group. ${ }^{\Delta} \mathrm{P}<0.05$ compared with the $\mathrm{DN}$ group. For group abbreviations, see legend to Table 1 . 


\section{DISCUSSION}

In the progression of diabetic nephropathy, the increased levels of radical products and the decreased activity of the antioxidant defense system can cause increased oxidative stress in the diabetic state (Roy et al., 2008), which will result in a vicious cycle. Antioxidants and vasoactive substances are able to enhance the body's antioxidant capacity by reducing the production of free radicals and directly quenching the radicals. A variety of antioxidants such as vitamins $\mathrm{C}$ and $\mathrm{E}$, lipoic acid, and vasoactive substances such as BPS have a beneficial effect on the complications of diabetic patients (Watanabe et al., 2009).

In this study, a high-fat diet with a small dose of intraperitoneal STZ was used to establish the T2DM rat model. These rats showed obesity, polydipsia, polyphagia, hyperglycemia, high islet hyperlipidemia, high triglycerides, hyperlipidemia, insulin resistance, and other characteristics. After given BPS for therapeutic intervention, the BPS rats showed improvement in symptoms of excessive drinking and eating. The body weight monitoring of rats in each group showed a progressive weight decline in the DN group with the development of the disease $(\mathrm{P}<0.01)$. There was no significant difference in body mass of the BPS group compared with the $\mathrm{NC}$ group $(\mathrm{P}>0.05)$. The poor glycemic control, the glucose metabolism disorders, the inability to make full use of glucose to supply energy, the acceleration of the decomposition of fat and protein to replenish their energy and heat are usually seen with duration of T2DM. As a result, carbohydrates, fat and protein are greatly consumed with the loss of moisture, and the mass of the patient's body will be significantly reduced (McKenney and Short, 2011). Blood glucose in the DN group was significantly higher compared with the NC group $(\mathrm{P}<0.01)$, while blood glucose was significantly lower in the BPS group compared with the $\mathrm{DN}$ group $(\mathrm{P}<0.01)$. Glucose metabolism in BPS rats significantly improved, so their body weight did not decrease with the progression of diabetes. In the early stage of diabetes, renal blood flow and glomerular filtration rate increases, and then, renal enlargement and microalbuminuria gradually occur. Due to the disorder of glucose metabolism and increased inflammatory response, hypoxia and hemodynamic changes in the kidney increase to result in the aggravation of renal microvascular disease and massive proteinuria, the glomerular filtration rate will gradually decline until renal failure (Rigalleau et al., 2011). In this study, 24-h urine, 24-h UAlb, Cr, and BUN significantly increased in the DN group compared with the NC group. The 24-h urine and $\mathrm{Cr}$ levels decreased slightly in the BPS group compared with the DN group $(\mathrm{P}<0.05), 24-\mathrm{h}$ UAlb significantly decreased in the BPS group $(\mathrm{P}<0.01)$, and there was no significant difference in BUN between the two groups $(\mathrm{P}>0.05)$. This study confirms that BPS can reduce urinary protein excretion in diabetic rats with proteinuria. To some extent, the treatment reverses the early glomerular pathological changes and provides a theoretical basis for clinical application.

In view of the theory that the chronic complications of diabetes share common ground (Nishikawa et al., 2000) and that their incidence are associated with oxidative stress, we should strive to protect the kidney by alleviating oxidative stress to the cells and tissues (Stanton, 2011). We found that the levels of the inflammatory indicators IL-6 and hs-CRP were significantly increased in the DN group compared with the NC group $(\mathrm{P}<0.01)$. The results showed that the oxidative stress response levels and the production of inflammatory indicators were significantly increased in the T2DM rats. hs-CRP levels significantly decreased in the BPS group compared with the DN group $(\mathrm{P}<0.01)$, and IL-6 levels decreased slightly in the BPS group $(\mathrm{P}<0.05)$. This study suggests that the BPS could reduce oxidative stress response 
levels, inhibit inflammatory factor production, improve endothelial function, and delay the onset of diabetic microangiopathy to finally reduce urinary protein excretion and protect renal function. During the progression of T2DM, oxidative stress levels are significantly elevated, inflammatory factors are generated, and endothelial function is increasingly impaired (Choi et al., 2008). A recent study by Sato et al. (2010) showed that BPS can improve impaired glucose tolerance and insulin resistance in obese rats, and that it can also reduce protein excretion. It can be speculated that the BPS treatment of diabetic complications such as diabetic nephropathy may result from improved glucose metabolism. Although the molecular mechanism of BPS is not clear, this study indicated that glucose metabolism, renal function and oxidative stress were significantly improved with BPS intervention in the T2DM rats.

In summary, this study provides a new way for the clinical treatment of patients with diabetic nephropathy.

\section{REFERENCES}

Brownlee M (2005). The pathobiology of diabetic complications: a unifying mechanism. Diabetes 54: 1615-1625.

Choi SW, Benzie IF, Ma SW, Strain JJ, et al. (2008). Acute hyperglycemia and oxidative stress: direct cause and effect? Free Radic. Biol. Med. 44: 1217-1231.

Gryglewski RJ (2008). Prostacyclin among prostanoids. Pharmacol. Rep. 60: 3-11.

McKenney RL and Short DK (2011). Tipping the balance: the pathophysiology of obesity and type 2 diabetes mellitus. Surg. Clin. North Am. 91: 1139-1148, vii.

Nishikawa T, Edelstein D, Du XL, Yamagishi S, et al. (2000). Normalizing mitochondrial superoxide production blocks three pathways of hyperglycaemic damage. Nature 404: 787-790.

Rigalleau V, Beauvieux MC, Gonzalez C, Raffaitin C, et al. (2011). Estimation of renal function in patients with diabetes. Diabetes Metab. 37: 359-366.

Roy M, Sen S and Chakraborti AS (2008). Action of pelargonidin on hyperglycemia and oxidative damage in diabetic rats: implication for glycation-induced hemoglobin modification. Life Sci. 82: 1102-1110.

Sato N, Kaneko M, Tamura M and Kurumatani H (2010). The prostacyclin analog beraprost sodium ameliorates characteristics of metabolic syndrome in obese Zucker (fatty) rats. Diabetes 59: 1092-1100.

Stanton RC (2011). Oxidative stress and diabetic kidney disease. Curr. Diab. Rep. 11: 330-336.

Watanabe M, Nakashima H, Mochizuki S, Abe Y, et al. (2009). Amelioration of diabetic nephropathy in OLETF rats by prostaglandin I(2) analog, beraprost sodium. Am. J. Nephrol. 30: 1-11. 